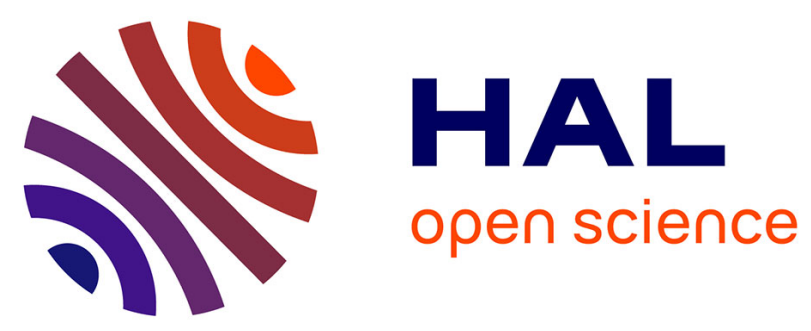

\title{
Towards a reduced in silico model predicting biochemical recurrence after radiotherapy in prostate cancer
}

Carlos Sosa-Marrero, Renaud de Crevoisier, Alfredo Hernandez, Pierre

Fontaine, Nathalie Rioux-Leclercq, Romain Mathieu, Alain Fautrel, Francois

Paris, Oscar Acosta

\section{To cite this version:}

Carlos Sosa-Marrero, Renaud de Crevoisier, Alfredo Hernandez, Pierre Fontaine, Nathalie RiouxLeclercq, et al.. Towards a reduced in silico model predicting biochemical recurrence after radiotherapy in prostate cancer. IEEE Transactions on Biomedical Engineering, 2021, 68 (9), pp.2718-2729. 10.1109/TBME.2021.3052345 . inserm-03137032

\section{HAL Id: inserm-03137032 https://www.hal.inserm.fr/inserm-03137032}

Submitted on 25 Feb 2021

HAL is a multi-disciplinary open access archive for the deposit and dissemination of scientific research documents, whether they are published or not. The documents may come from teaching and research institutions in France or abroad, or from public or private research centers.
L'archive ouverte pluridisciplinaire HAL, est destinée au dépôt et à la diffusion de documents scientifiques de niveau recherche, publiés ou non, émanant des établissements d'enseignement et de recherche français ou étrangers, des laboratoires publics ou privés. 


\title{
Towards a reduced in silico model predicting biochemical recurrence after radiotherapy in prostate cancer
}

\author{
Carlos Sosa-Marrero, Renaud de Crevoisier, Alfredo Hernández, Pierre Fontaine, Nathalie Rioux-Leclercq, \\ Romain Mathieu, Alain Fautrel, François Paris and Oscar Acosta
}

\begin{abstract}
Objective: Purposes of this work were i) to develop an in silico model of tumor response to radiotherapy, (ii) to perform an exhaustive sensitivity analysis in order to iii) propose a simplified version and iv) to predict biochemical recurrence with both the comprehensive and the reduced model. Methods: A multiscale computational model of tumor response to radiotherapy was developed. It integrated the following radiobiological mechanisms: oxygenation, including hypoxic death; division of tumor cells; VEGF diffusion driving angiogenesis; division of healthy cells and oxygen-dependent response to irradiation, considering, cycle arrest and mitotic catastrophe. A thorough sensitivity analysis using the Morris screening method was performed on 21 prostate computational tissues. Tumor control probability (TCP) curves of the comprehensive model and 15 reduced versions were compared. Logistic regression was performed to predict biochemical recurrence after radiotherapy on $\mathbf{7 6}$ localized prostate cancer patients using an output of the comprehensive and the reduced models. Results: No significant difference was found between the TCP curves of the comprehensive and a simplified version which only considered oxygenation, division of tumor cells and their response to irradiation. Biochemical recurrence predictions using the comprehensive and the reduced models improved those made from pre-treatment imaging parameters $(\mathrm{AUC}=\mathbf{0 . 8 1} \pm$ 0.02 and $0.82 \pm 0.02$ vs. $0.75 \pm \mathbf{0 . 0 3}$, respectively). Conclusion: A reduced model of tumor response to radiotherapy able to predict biochemical recurrence in prostate cancer was obtained. Significance: This reduced model may be used in the future to optimize personalized fractionation schedules.
\end{abstract}

Index Terms-Biochemical recurrence, multiscale modeling, prostate cancer, radiotherapy, sensitivity analysis, tumor control probability

\section{INTRODUCTION}

$\mathbf{E}$ XTERNAL beam radiotherapy (EBRT) is the most common treatment for localized prostate cancer. A total dose between 70 and $80 \mathrm{~Gy}$ is typically prescribed and delivered in several fractions during 7 or 8 weeks from Monday to Friday, in order to allow healthy tissues to recover while preventing tumor from complete repair [1]. Tumor local control is achieved in $60 \%$ to $90 \%$ of cases, depending on the D'Amico prostate

This work was supported by the Brittany Region and FHU TECH-SAN.

C. Sosa-Marrero, R. de Crevoisier, A. Hernández, P. Fontaine, and O. Acosta are with Univ Rennes, CLCC Eugène Marquis, INSERM, LTSI - UMR 1099, F-35000 Rennes, France (correspondence e-mail: carlos.sosa@univrennes1.fr). N. Rioux-Leclercq and R. Mathieu are with Univ Rennes, CHU Rennes, Inserm, EHESP, Irset - UMR-S 1085, F-35000 Rennes, France. A. Fautrel is with Univ Rennes, CNRS, Inserm, UMS Biosit, Biogenouest, Core Facility H2P2, F-35000 Rennes, France. F. Paris is with CRCINA, INSERM, UMR 1232, CNRS, Univ Nantes, France. cancer risk group [2]. The conception of modified patientspecific therapies that may help to increase these percentages is hindered by the complexity of the biological mechanisms characterizing cancer and the response to irradiation of both tumor and surrounding healthy tissues.

Numerous radiobiological mechanisms have been considered to participate in tumor survival after radiotherapy and later recurrence. In particular, those related to the 5 R's (reoxygenation, repopulation, DNA repair, radiosensitivity and redistribution in the cell cycle) have been thoroughly studied [3]. Modulation of tumor radiation resistance is thought to be related, at least, to hypoxia and abnormal angiogenesis resulting in the reoxygenation of the tissue [4]-[6], a high rate of proliferation/repopulation of tumor cells [7]-[9] and a low intrinsic radiosensitivity of tumor cells [10]-[12], associated with a preponderant cell cycle distribution in the radioresistant phase S [13]. Mitotic catastrophe [14], considered to be the main type of cell death after irradiation, may also play a major role in tumor control [15], [16].

In silico modeling [17], [18] emerges as a powerful tool to integrate these radiobiological mechanisms and predict their behavior on hypothetical scenarios. Computational models make it possible to create, at a limited cost, infinite virtual tumors with different vascular architectures on which various irradiation schedules can be simulated. The long-term aim is to personalize the decision making process in the radiotherapy workflow.

According to their spatial scale, in silico models can be classified into 3 categories. Microscopic models [19], [20] consider the stochastic behavior of individual cells and the interactions between them. They can reflect cell heterogeneity but may not reproduce a realistic tumor microenvironment and the link to the image is not clear. Harting et al. [20] proposed a single-cell-based model that integrated tumor proliferation, oxygenation and radiation response. It also included angiogenesis, considering the diffusion of tumor angiogenesis factors (TAF) emitted by hypoxic cells.

Macroscopic models [21]-[25], in contrast, describe spatiotemporal changes in tumor cell density using differential equations at the tissue scale, which makes them susceptible to be validated with clinical data. Belfatto et al. [24] proposed a computational model based on Gompertzian growth and fitted with uterine cervical cancer CBCT scans. It included oxygenation and a response to irradiation that considered both its instantaneous effect and its delayed cell killing capability, 
which may be associated with mitotic catastrophe.

Hybrid or multiscale models arise to combine the advantages of the 2 previous approaches in a single framework. Numerous multiscale in silico models of tumor response to radiotherapy already exist in the literature. For the vast majority of them, the response to irradiation is based upon the widely-used linear-quadratic formalism [26]-[28]. Titz et al. [29] developed a model of tumor growth and response to radiation that incorporated cell cycle distribution. This allowed to consider phase-dependent radiosensitivity. Espinoza et al. [30] proposed a voxel-based multiscale model to simulate the radiation response of hypoxic tumors. Precalculated oxygen histograms for each voxel were used to simulate hypoxicinduced angiogenesis and oxygen-dependent response to irradiation. Paul-Gilloteaux et al. [31] developed a 2D and 3D cellular automaton that was validated on human prostate tumors transplanted in mice and then used to generate tumor control probability (TCP) curves for different radiotherapy protocols. It introduced mitotic catastrophe instead of arbitrary delayed cell death. Apeke et al. [32] proposed another in silico model considering cell cycle distribution at a mesoscopic scale. Realistic static oxygenation maps were obtained from FDG PET images.

Several issues are intrinsic to multiscale modeling. Firstly, multiscale models integrate complex radiobiological mechanisms occurring at different spatial and temporal scales (from cell to tissue and from milliseconds to months, respectively). This constitutes a serious challenge from a mathematical and computational point of view. Simulations must have adapted time-steps and spatial resolutions in order to capture changes while limiting redundant iterations. They must be synchronized at a reasonable frequency to be able to reflect the numerous interactions existing among them. Computational libraries like M2SL (Multi-formalism Modeling and Simulation Library) [33] have been developed to facilitate the integration of different multiscale processes in the modeling task.

Secondly, hybrid models may contain a large number of parameters. Some of them are difficult to measure in vivo or even in vitro, which hampers model calibration and validation. Sensitivity analysis can be used to study the impact of all the parameters of a model on a given output, identify the most relevant ones and determine which ones could be negligible [34], [35]. Variance-based analysis, like the Sobol method [36], give a quantitative expression of the importance of all the parameters of a model. However, they are computationally expensive. Screening analysis, like the Morris method [37], provide a qualitative view of the hierarchy of parameters with a reduced computational cost. Multiple examples of sensitivity analysis allowing subsequent parameter identification and model dimension reduction can be found in the biomedical literature [38]-[40].

Overall, a large number of in silico models combining some of the most important biological processes characterizing cancer and the response to radiotherapy have already been proposed in the literature. However, some of these mechanisms, which may play a major role in tumor control, have never been included in a single comprehensive model. In particular, no work of the literature has simultaneously integrated, to our knowledge, dynamic oxygenation, mitotic catastrophe and cell cycle distribution allowing the simulation of a phasedependent radiosensitivity for tumor cells. Moreover, although the independent contribution of different radiobiological mechanisms has been evaluated for some of the models through TCP or tumor density curves [20], [30], [31], a thorough sensitivity analysis to precisely identify the impact of each radiobiological parameter has not been performed yet for any of the models.

Objectives of the present work were: i) to develop a comprehensive in silico model of tumor response to radiotherapy integrating the major radiobiological mechanisms, taking place at different spatial and temporal scales, ii) to perform a thorough sensitivity analysis of the model in order to reduce the number of parameters and iii) propose a more compact equivalent version and finally, iv) to demonstrate its clinical usefulness in prediction of biochemical recurrence after prostate cancer radiotherapy. A preliminary version of this work has been reported [41].

The paper is structured as follows: in section II, the model of tumor response to radiotherapy, the principles of the Morris screening method and the reduction and validation experiments are described. The results of the comprehensive model, the sensitivity analysis, the reduced version and the preliminary validation are presented and discussed in sections III and IV, respectively. Finally, conclusions are drawn in section V.

\section{Material And methods}

\section{A. Description of the model of tumor response to radiotherapy}

1) General description: The in silico model of tumor response to radiotherapy considered either a $2 \mathrm{D}$ or a $3 \mathrm{D}$ prostate computational tissue (Fig. 2) where each pixel (20 $\mu \mathrm{m} \times 20 \mu \mathrm{m})$ or voxel $(20 \mu \mathrm{m} \times 20 \mu \mathrm{m} \times 20 \mu \mathrm{m})$ corresponded to a cell of one of the following 6 types: healthy (fibroblasts, macrophages, epithelial, smooth muscle, etc.), undamaged or lethally damaged tumor, pre-existing or neocreated endothelial and dead.

The model considered the following radiobiological mechanisms, happening at different temporal and spatial scales: (i) oxygenation of the tissue, (ii) division of tumor cells, (iii) angiogenesis, (iv) division of healthy cells and (v) response to irradiation. A functional diagram of the model is presented in Fig. 1. The model was implemented in $\mathrm{C}++$ based upon the Multiformalism Modeling and Simulation Library (M2SL) [33]. This in-house library previously developed in other biomedical modeling contexts [42], allows the integration of different processes arising at different temporal and spatial scales expressed with various formalisms (algebraic equations, partial differential equations and cell automata). In this work, we extended the functionalities to 2D and 3D arrays allowing fast simulations of multiple simultaneous processes. Further details about model implementation can be found in supplementary material.

2) Oxygenation (Reoxygenation): Oxygenation of the tissue was modeled, as in [43], using the reaction-diffusion equation (1)

$$
\frac{\partial u(\boldsymbol{x}, t)}{\partial t}=D^{O_{2}} \Delta u(\boldsymbol{x}, t)-r(u(\boldsymbol{x}, t))
$$

\section{ACCEPTED MANUSCRIPT}




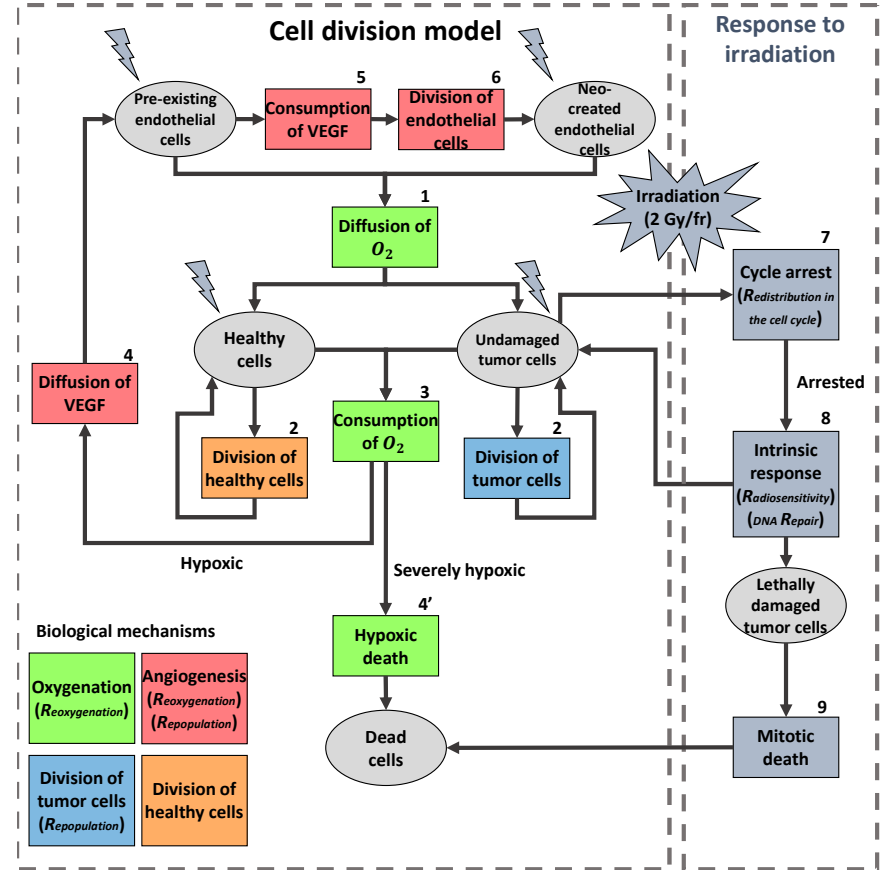

Fig. 1. Functional diagram of the model. The different types of simulated cells are represented with a gray ellipse. The different mechanisms are represented with a rectangle. The same color code as in Table I is used. Correspondences to the $5 \mathrm{R}$ 's are indicated in italics. Endothelial cells diffuse $\mathrm{O}_{2}(1)$. Healthy and tumor cells divide (2) and consume $\mathrm{O}_{2}$ (3). As a result, they may become hypoxic, in which case they diffuse VEGF (vascular endothelial growth factor) (4), or, if the $\mathrm{O}_{2}$ levels are extremely low, severely hypoxic, which provokes their death (4'). VEGF is consumed by endothelial cells (5), resulting in their division (6). Tumor cells irradiated with a dose of 2 Gy per fraction are arrested (7). According to their intrinsic response to radiation (8), if their DNA can be repaired, they resume their division. If the damage caused by irradiation is lethal, they die at the next mitosis (mitotic death) (9).

where $u(\boldsymbol{x}, t)$ is the oxygen concentration; $D^{O_{2}}$, the oxygen diffusion coefficient and $r(u(\boldsymbol{x}, t))$, the oxygen consumption, calculated, for healthy and tumor cells, with the MichaelisMenten equation (2)

$$
r(u(\boldsymbol{x}, t))=V_{\max }^{O_{2}} \frac{u(\boldsymbol{x}, t)}{u(\boldsymbol{x}, t)+K_{M}^{O_{2}}}
$$

where $V_{\text {max }}^{O_{2}}$ is the maximum oxygen consumption ratio and $K_{M}^{\mathrm{O}_{2}}$, the Michaelis constant. Dead cells were considered to consume no oxygen.

Henry's law states that, at a constant temperature, the amount of gas dissolved in a liquid is proportional to the partial pressure that the gas applies to the liquid (3),

$$
p=K_{H} u,
$$

with $p$, the partial pressure and $K_{H}$, the Henry's constant that depends on the gas, the liquid and the temperature. Using this relationship and redefining $K_{M}^{O_{2}}$ as $K_{M}^{O_{2}}:=K_{M}^{O_{2}} K_{H}$ and $V_{\max }^{\mathrm{O}_{2}}$ as $V_{\max }^{\mathrm{O}_{2}}:=V_{\max }^{\mathrm{O}_{2}} K_{H}$, the oxygenation of the tissue could be expressed as a function of the partial pressure of oxygen (4) [43]

$$
\frac{\partial p O_{2}(\boldsymbol{x}, t)}{\partial t}=D^{O_{2}} \Delta p O_{2}(\boldsymbol{x}, t)-r\left(p O_{2}(\boldsymbol{x}, t)\right),
$$

with $r\left(p O_{2}(\boldsymbol{x}, t)\right)$ calculated for healthy and tumor cells as (5)

$$
r\left(p O_{2}(\boldsymbol{x}, t)\right)=V_{\max }^{O_{2}} \frac{p O_{2}(\boldsymbol{x}, t)}{p O_{2}(\boldsymbol{x}, t)+K_{M}^{O_{2}}} .
$$

Pre-existing and neo-created endothelial cells were supposed to have fixed $p O_{2}$ values $p O_{2}^{p r e E n d}$ and $p O_{2}^{\text {neoEnd }}$, respectively. The steady state solution was obtained numerically using the finite differences method (see section A in supplementary material for further details). Healthy and tumor cells having a $p \mathrm{O}_{2}$ lower than a threshold, $p \mathrm{O}_{2}^{\text {nec }}$, were considered to die instantaneously due to hypoxia.

3) Division of tumor cells (Repopulation): The tumor cell cycle was implemented like in the models proposed in [29] and [32]. It had a duration $T_{\text {tum }}$ and was composed of 4 phases: G1 (gap 1), S (synthesis), G2 (gap 2) and M (mitosis). In our model, there existed a fifth phase, called G0 and placed out of the cycle, in which cells were quiescent. When an undamaged tumor cell arrived at the end of its cycle, it divided, replacing a healthy or dead cell of its Moore neighborhood of order $N$ with a new tumor cell. If there was no adjacent healthy or dead cell, then it entered the phase G0.

Tumor cells with no available place in their Moore neighborhood were initialized in phase G0. Cycling cells were supposed to be distributed at the beginning of the simulation according to the following percentages: $60 \%$ in phase G1; $25 \%$, in $\mathrm{S} ; 7.5 \%$, in $\mathrm{G} 2$ and $7.5 \%$, in $\mathrm{M}$ [29].

4) Angiogenesis (Reoxygenation, repopulation): The model of angiogenesis was based on the VEGF (vascular endothelial growth factor) diffusion. This protein, consumed by endothelial cells, is emitted by hypoxic cells to provoke the creation of blood vessels that satisfy their oxygen needs. The VEGF distribution was assumed to be given by the reaction-diffusion equation (6)

$$
\frac{\partial v(\boldsymbol{x}, t)}{\partial t}=D^{V E G F} \Delta v(\boldsymbol{x}, t)-r(v(\boldsymbol{x}, t)),
$$

where $D^{V E G F}$ is the diffusion coefficient and $r(v(\boldsymbol{x}, t))$, the VEGF consumption, calculated, for endothelial cells, with the Michaelis-Menten equation

$$
r(v(\boldsymbol{x}, t))=V_{\max }^{V E G F} \frac{v(\boldsymbol{x}, t)}{v(\boldsymbol{x}, t)+K_{M}^{V E G F}},
$$

where $V_{\max }^{V E G F}$ is the maximum VEGF consumption ratio and $K_{M}^{V E G F}$, the Michaelis constant. Hypoxic cells (with a $p \mathrm{O}_{2}$ value lower than a given threshold, $p O_{2}^{h y p}$ ) were supposed to have a fixed $v$ value, $v^{\text {hyp }}$ [20]. The finite differences method was used to obtain the numerical steady state solution (see section A in supplementary material for further details). A cycle duration, $T_{\text {end }}$, was defined for both pre-existing and neo-created endothelial cells. If at the end of its cycle, the VEGF concentration of an endothelial cell whose DNA had not been lethally damaged by irradiation exceeded a predetermined value $\bar{v}$, the cell in question divided. If not, it entered the quiescent phase G0, where it remained until a potential augmentation of $v(x, t)$. The new endothelial cell was placed in the most hypoxic direction [20]. 
5) Division of healthy cells: The duration of the healthy cell cycle $T_{\text {heal }}$ was defined. When a cell whose DNA had not been lethally damaged by irradiation arrived at the end of its cycle, it divided, replacing a dead cell of its Moore neighborhood of order $N$ with a new healthy cell. If there was no adjacent dead cell, then it entered the phase G0.

6) Response to irradiation (Redistribution in the cell cycle, radiosensitivity, DNA repair): The response of every cell to irradiation was modeled as the survival fraction (SF) following the linear-quadratic equation and adjusted to consider the influence of the $\mathrm{pO}_{2}$ (8) [20], [29]-[31]

$$
S F=\exp \left(-\frac{\alpha}{m} d O E R\left(p O_{2}\right)-\frac{\beta}{m^{2}} d^{2} O E R^{2}\left(p O_{2}\right)\right),
$$

where $\alpha$ and $\beta$ are the radiosensitivity parameters; $d$, the administered dose per session and $O E R$, the oxygen enhancement ratio given by (7)

$$
\operatorname{OER}\left(p \mathrm{O}_{2}\right)=\frac{m p O_{2}+k}{p O_{2}+k},
$$

with $m$, the maximum value of the $O E R$ (set to 3 [15]) and $k$, the partial pressure of oxygen such that $O E R=(m+1) / 2$ $(3 \mathrm{mmHg})$. The radiosensitivity of tumor cells was supposed to vary throughout the cycle. Thus, cells were more sensitive in phases $\mathrm{G} 2$ and $\mathrm{M}$ and more resistant to irradiation in phase S [13]. Healthy and endothelial cells, significantly more radioresistant, had constant values of $\alpha$ and $\beta$ throughout their cycle. Irradiated cells were arrested for a duration $T_{\text {arrest }}$ at checkpoints located in transitions between phases G1/S and G2/M [31]. Cells lethally damaged by irradiation died by mitotic catastrophe.

\section{B. Morris screening method}

The Morris screening method [37] offers an overall view of the influence of the parameters $\boldsymbol{x}=x_{1}, \ldots, x_{K}$ of a model $\mathbf{y}=f(\boldsymbol{x})$ on its outputs $\boldsymbol{y}=y_{1}, . ., y_{L}$ with a low computational cost. Additionally, it provides information about the nature of the impact (linear or non-linear or having interactions with other parameters). It explores a $K$-dimensional cube regularly divided in $p$ levels. In this space, for an output $y_{j}$, $N$ elementary effects, given by (10) are calculated for each factor $x_{i}$. A clever experimental plan taking $\Delta$, the discrete variation of the parameter, equal to $\frac{p}{2(p-1)}$ with $p$ even, is used. It requires, in total, $N(K+1)$ evaluations of the model.

$$
E E_{i}=\frac{f\left(x_{1}, \ldots, x_{i}, \ldots, x_{K}\right)-f\left(x_{1}, \ldots, x_{i}+\Delta, \ldots, x_{K}\right)}{\Delta} .
$$

The mean and standard deviation $\left(\mu_{i}^{*} \pm \sigma_{i}\right)$ over the absolute values [44] of the elementary effects are computed for each parameter. The Euclidean distance of each point $\left(\mu_{i}^{*}, \sigma_{i}\right)$ to the origin, $S_{i}=\sqrt{\mu_{i}^{* 2}+\sigma_{i}^{2}}$, can be calculated as an indicator of the impact of the parameter in question [39]. The prostate-specific ranges presented in Table I were considered for the $K=33$ parameters of the model. They were obtained calculating, respectively, 0.7 and 1.3 of the minimum $P^{\text {min }}$ and maximum $P^{\max }$ values extracted from the literature or our radiobiological expertise. Ranges of the angiogenesis parameters were based on the global results of our previous study of vasculature in orthotopic mouse prostate cancer [45].

\section{Tumor control probability curves}

Tumor control probability (TCP) curves, typically used in clinical trials [1], [50], were generated as virtual endpoints in the simulations. A tumor was supposed to be controlled when the computational tissue did not contain any undamaged tumor cell [20], [31]. Using this definition, TCP curves can be fitted by the sigmoid function (11)

$$
T C P(D)=\frac{1}{1+\exp (-a(D-b))},
$$

where $D$ is the total dose; $a$, the tangent at the inflection point and $b$, its shift. It can be noticed that a value of $D$ equal to $b$ leads to a $T C P$ of $50 \%\left(T C P_{50}\right)$. Thus, $b$ can be interpreted as the dose necessary to have a tumor control probability of $50 \%\left(T C D_{50}\right)$.

\section{Simplification of the model}

Based on the results of the sensitivity analysis, the initial comprehensive model was contrasted, through TCP curves, with simplified versions where different mechanisms or submechanisms were progressively removed. Firstly, the complete model was compared with 4 reduced versions that did not consider angiogenesis, healthy cell division, cycle arrest or the response to irradiation of healthy and endothelial cells. Then, the differences between the comprehensive model and simplified versions that did not include 2-and-3-element combinations of these mechanisms were studied. Finally, the complete model was contrasted with a reduced version that integrated neither angiogenesis, nor healthy cell division nor cycle arrest, nor the response to irradiation of healthy and endothelial cells.

\section{E. Initialization of the model from prostate histological cuts}

In order to perform simulations on realistic configurations of tumor and vascular cells, HES and CD31 prostate histological cuts from 7 patients treated with radical prostatectomy were used to initialize the model for the sensitivity analysis and the simplification experiments. Tumor foci were delineated on the HES axial slides (Fig. 3a) and a CD31 staining (Fig. 3b) was carried out to identify the blood vessels. Twenty-one regions of interest (ROI) of approximately $2 \mathrm{~mm} \times 1.2 \mathrm{~mm}$ (100 pixels $\times 60$ pixels) were selected from the tumor foci to create 21 initial computational tissues with different tumor and vascular densities. An example of ROI and the corresponding virtual tissue are presented in Fig. 3c and d, respectively.

\section{F. Validation of the model. Biochemical recurrence prediction}

A first clinical validation of both the comprehensive and the reduced model in terms of biochemical recurrence prediction was performed. A cohort of 76 localized prostate cancer patients having undergone EBRT [51] was used for this purpose. Patient, tumor and treatment characteristics are presented in Supplementary Table I. Patients were followed up by means

\section{ACCEPTED MANUSCRIPT}




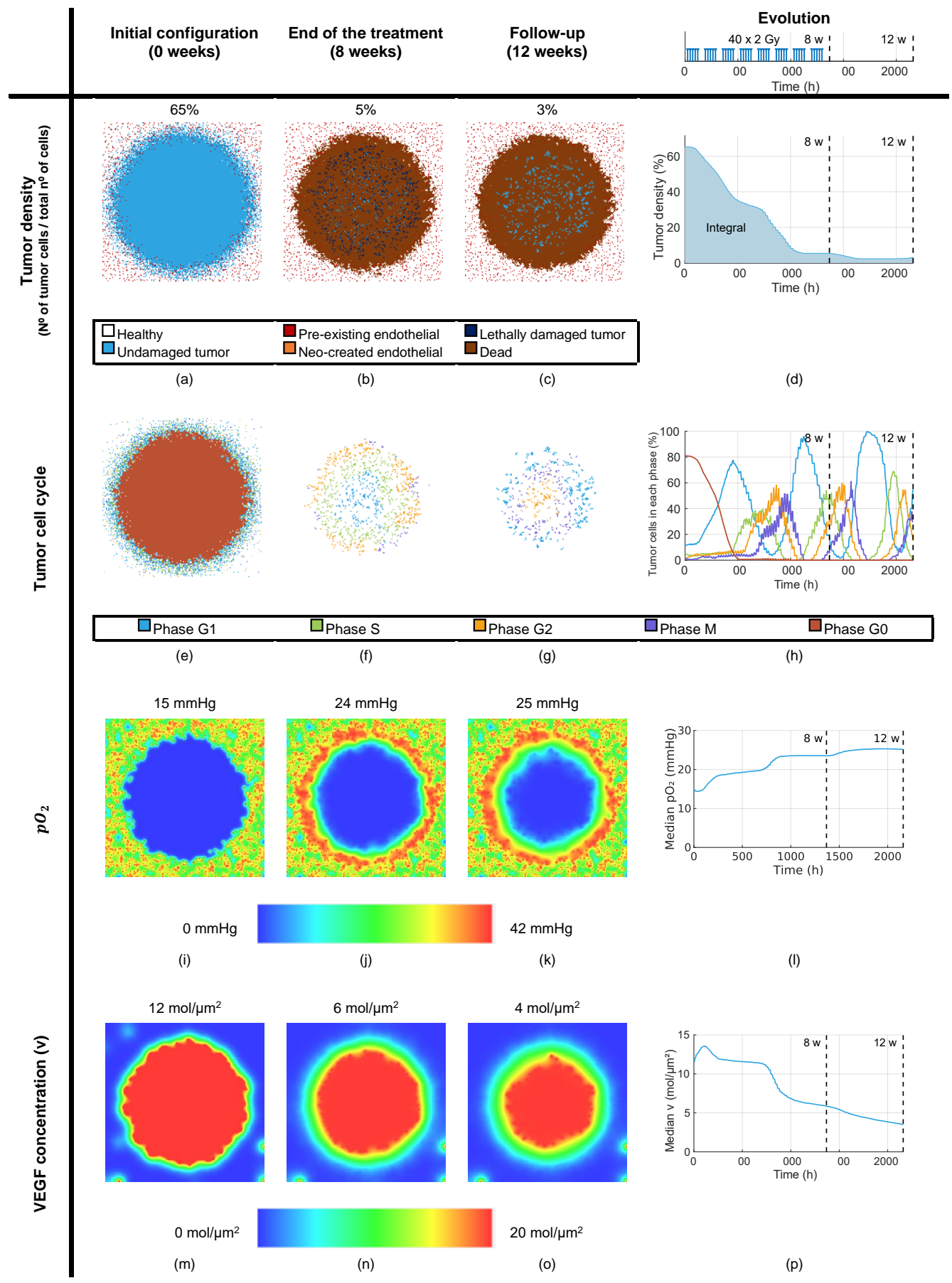

Fig. 2. Example of a $40 \times 2$ Gy treatment on a synthetic tissue. Tumor density at (a) the beginning of the simulation $(t=0$ weeks); (b) the end of a $40 \times$ 2 Gy treatment ( $t=8$ weeks) and (c) $t=12$ weeks after the beginning of the treatment; (d) evolution of tumor density (the integral of tumor density can be deduced); corresponding distribution of tumor cells in the cycle at (e) $t=0$ weeks, (f) $t=8$ weeks and (g) $t=12$ weeks; (h) evolution of the distribution of tumor cells in the cycle; $\mathrm{pO}_{2}$ map at (i) $t=0$ weeks; (j) $t=8$ weeks and (k) $t=12$ weeks; (l) evolution of the median $\mathrm{pO}_{2}$ of the tissue; VEGF concentration map at (m) $t=0$ weeks; (n) $t=8$ weeks and (o) $t=12$ weeks and (p) evolution of the median VEGF concentration of the tissue.

of clinical examination and PSA analysis every 6 months for 5 years after the end of irradiation. Nine patients suffered biochemical recurrence, defined according to the Phoenix criteria [52]. Our IRB approved this retrospective study.

The logistic model (stratified 3-fold cross-validation, 1000 repetitions) was used to predict biochemical recurrence. A first prediction was made from 3 pre-treatment imaging parameters, namely maximal tumor area and average ADC and $\mathrm{T} 2 \mathrm{w}$, obtained from 3.0 T MRI.

Biochemical recurrence was then predicted using the tumor

\section{ACCEPTED MANUSCRIPT}


TABLE I

RANGES AND REFERENCE VALUES OF THE 33 PARAMETERS OF THE COMPREHENSIVE MODEL, CLASSIFIED BY RADIOBIOLOGICAL MECHANISM

\begin{tabular}{|c|c|c|}
\hline \multicolumn{3}{|c|}{ Oxygenation } \\
\hline Factor & Range & Reference value \\
\hline$p O_{2}^{\text {nec }}(\mathrm{mmHg})$ & $0-1.3[29],[31]$ & 0.7 \\
\hline$D^{O_{2}}\left(\mu \mathrm{m}^{2} / \mathrm{ms}\right)$ & $1.02-2.87[43]$ & 1.84 \\
\hline$V_{\max }^{O_{2}}(\mathrm{mmHg} / \mathrm{ms})$ & $0.006-0.029[43]$ & 0.015 \\
\hline$K_{M}^{O_{2}}(\mathrm{mmHg})$ & $0.119-7.67[43]$ & 3.04 \\
\hline$p O_{2}^{\text {preEnd }}(\mathrm{mmHg})$ & $8.4-93.6[46]$ & 42 \\
\hline$p O_{2}^{\text {neoEnd }}(\mathrm{mmHg})$ & $8.4-93.6[46]$ & 42 \\
\hline
\end{tabular}

(a)

\begin{tabular}{|c|c|c|}
\hline \multicolumn{3}{|c|}{ Division of tumor cells } \\
\hline Factor & Range & Reference value \\
\hline$T_{\text {tum }}(\mathrm{h})$ & $85-1310[30],[47]$ & 565 \\
\hline$N$ & $1-3[31]$ & 1 \\
\hline
\end{tabular}

(b)

\begin{tabular}{|c|c|c|}
\hline \multicolumn{3}{|c|}{ Angiogenesis } \\
\hline Factor & Range & Reference value \\
\hline$T_{\text {end }}(\mathrm{h})$ & $1680-3120$ & 2400 \\
\hline$D^{V E G F}\left(\mu \mathrm{m}^{2} / \mathrm{ms}\right)$ & $1.4-2.6$ & 2 \\
\hline$V_{\max }^{V E G F}\left(\mathrm{~mol} / \mu \mathrm{m}^{2} \mathrm{~ms}\right)$ & $0.005-0.007$ & 0.006 \\
\hline$K_{M}^{V E G F}\left(\mathrm{~mol} / \mu \mathrm{m}^{2}\right)$ & $1.75-3.25$ & 2.5 \\
\hline$p O_{2}^{h y p}(\mathrm{mmHg})$ & $3.5-6.5[31]$ & 5 \\
\hline $\bar{v}\left(\mathrm{~mol} / \mu \mathrm{m}^{2}\right)$ & $10.5-19.5$ & 15 \\
\hline$v^{h y p}\left(\mathrm{~mol} / \mu \mathrm{m}^{2}\right)$ & $14-26$ & 20 \\
\hline
\end{tabular}

(c)

\begin{tabular}{|c|c|c|}
\hline \multicolumn{3}{|c|}{ Division of healthy cells } \\
\hline Factor & Range & Reference value \\
\hline$T_{\text {heal }}(\mathrm{h})$ & $171-2620$ & 1130 \\
\hline
\end{tabular}

(d)

\begin{tabular}{|c|c|c|}
\hline \multicolumn{3}{|c|}{ Response to irradiation } \\
\hline Factor & Range & Reference value \\
\hline$\alpha_{\text {heal }}\left(\mathrm{Gy}^{-1}\right)$ & $7 \cdot 10^{-4}-1.3 \cdot 10^{-3}$ & 0.001 \\
\hline$\alpha / \beta_{\text {heal }}(\mathrm{Gy})$ & $0.7-13$ & 5.5 \\
\hline$\alpha_{t u m G 1}\left(\mathrm{~Gy}^{-1}\right)$ & $0.024-0.356$ [48], [49] & 0.154 \\
\hline$\alpha / \beta_{t u m G 1}(\mathrm{~Gy})$ & $0.7-13$ [48], [49] & 5.5 \\
\hline$\alpha_{t u m S}\left(\mathrm{~Gy}^{-1}\right)$ & $0.017-0.256$ [48], [49] & 0.111 \\
\hline$\alpha / \beta_{\text {tumS }}(\mathrm{Gy})$ & $0.7-13$ [48], [49] & 5.5 \\
\hline$\alpha_{\text {tumG2 }}\left(\mathrm{Gy}^{-1}\right)$ & $0.025-0.381$ [48], [49] & 0.165 \\
\hline$\alpha / \beta_{G 2}(\mathrm{~Gy})$ & $0.7-13$ [48], [49] & 5.5 \\
\hline$\alpha_{t u m M}\left(\mathrm{~Gy}^{-1}\right)$ & $0.028-0.425$ [48], [49] & 0.184 \\
\hline$\alpha / \beta_{t u m M}(\mathrm{~Gy})$ & $0.7-13$ [48], [49] & 5.5 \\
\hline$\alpha_{t u m G 0}\left(\mathrm{~Gy}^{-1}\right)$ & $0.105-0.195$ [48], [49] & 0.15 \\
\hline$\alpha / \beta_{\text {tumG0 }}(\mathrm{Gy})$ & $0.7-13$ [48], [49] & 5.5 \\
\hline$\alpha_{\text {preEnd }}\left(\mathrm{Gy}^{-1}\right)$ & $7 \cdot 10^{-4}-1.3 \cdot 10^{-3}[31]$ & 0.001 \\
\hline$\alpha / \beta_{\text {preEnd }}(\mathrm{Gy})$ & $0.7-13[31]$ & 5.5 \\
\hline$\alpha_{n e o E n d}\left(\mathrm{~Gy}^{-1}\right)$ & $7 \cdot 10^{-4}-1.3 \cdot 10^{-3}[31]$ & 0.001 \\
\hline$\alpha / \beta_{\text {neoEnd }}(\mathrm{Gy})$ & $0.7-13[31]$ & 5.5 \\
\hline$T_{\text {arrest }}(\mathrm{h})$ & $4.2-39$ & 18 \\
\hline
\end{tabular}

(e)

Parameters ranges, used for the sensitivity analysis, were extracted from the literature and our radiobiological expertise. Reference values were defined as intermediate values within the ranges.

area at $t=8$ weeks output given by the comprehensive and

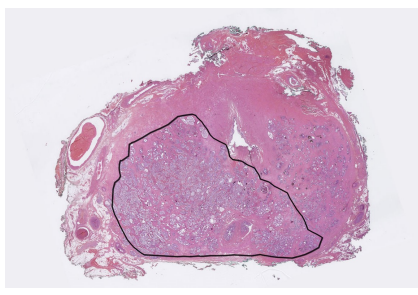

(a)

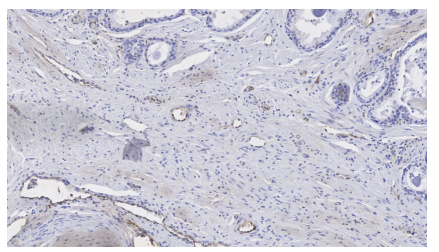

(c)

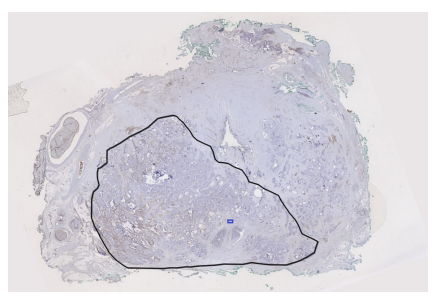

(b)

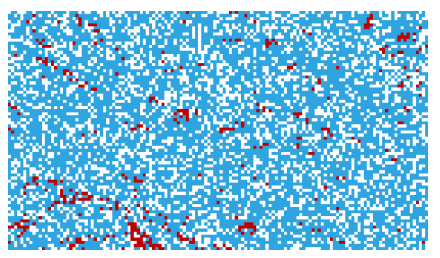

(d)
Fig. 3. Example of (a) HES staining of a prostate histological cut with tumor focus delineated by an anatomopathologist; (b) corresponding CD31 staining; (c) CD31 staining region of interest where vessels can be identified in brown and (d) initial computational tissue, where healthy cells are represented in white; undamaged tumor, in light blue and pre-existing endothelial, in red.

reduced in silico models. For this purpose, 76 virtual tissues analogous to the 76 patients of the cohort were built from their respective average $\mathrm{ADC}$ and $\mathrm{T} 2 \mathrm{w}$ values, correlated with cell density [53], and maximal tumor areas observed before treatment. The radiotherapy protocol administered to each patient of the cohort (a total dose of $74-80$ Gy delivered in 2 Gy fractions from Monday to Friday) was then simulated on the corresponding virtual tissue. Reference values given in Table I were taken for every parameter except for the highly influential hypoxic death threshold $\mathrm{pO}_{2}^{\text {nec }}$, for which a low value was fixed. An initial vascular density of $3.8 \%$ [31], within the ranged observed in our histological cuts, was considered for every virtual tissue. Endothelial cells were supposed to be randomly distributed forming a poorlyvascularized tumor core. Given the stochastic component of the model, each simulation was repeated 5 times and the mean output value was taken.

\section{RESULTS}

\section{A. Integrative model of tumor response to radiotherapy}

As a proof of concept, a $40 \times 2$ Gy treatment, typically applied in prostate cancer, was tested on a synthetic tissue. Results are presented in Fig. 2. Tumor density, cell cycle, $p O_{2}(x, t)$ and $v(x, t)$ maps at the beginning of the simulation ( $t=0$ weeks), $t=8$ weeks (end of the treatment) and $t$ $=12$ weeks are shown. Curves of tumor density, cell cycle distribution and median $p \mathrm{O}_{2}$ and $v$ evolution through time are also presented.

\section{B. Sensitivity analysis}

The sensitivity analysis using the Morris method was performed on the 21 computational tissues obtained from the prostate histological cuts, considering a treatment of $40 \times$ 2 Gy, administered every $24 \mathrm{~h}$ from Monday to Friday. The 
tumor density 12 weeks after the beginning of the treatment and its integral over time were used as endpoints. The value of $\Delta$, considering $p=20$, was normalized for each parameter as $\frac{\Delta}{1.3 P^{\max }-0.7 P^{\min }}$. In order to ensure convergence, $N=100$ was taken. A total of $21 \times 100 \times(33+1)=71400$ simulations were thus performed. The Euclidean distances $S_{i}$ of the 33 parameters of the complete model for the 2 outputs considered are presented in Fig. 4.

The duration of the tumor cell cycle, $T_{t u m}$, and the radiosensitivity parameter of tumor cells in the phase G1, $\alpha_{t u m G 1}$, were identified as the factors having the highest impact on the tumor density at $t=12$ weeks (Fig. 4a). Other radiosensitivity parameters of tumor cells $\left(\alpha_{t u m G 2}, \alpha / \beta_{t u m G 1}, \alpha_{t u m M}\right.$ and $\alpha_{\text {tumS }}$ ) and oxygenation factors, like $p O_{2}^{\text {nec }}, K_{M}^{\mathrm{O}_{2}}, p \mathrm{O}_{2}^{\text {preEnd }}$ and $V_{m a x}^{O_{2}}$, had a considerable impact on this output as well.

$T_{\text {tum }}$ was also the most influential parameter on the integral of tumor density (Fig. 4b). The hypoxic death threshold, $p O_{2}^{n e c}$, had the second most important effect. Other factors of the oxygenation mechanism, like $p \mathrm{O}_{2}^{\text {preEnd }}, \mathrm{V}_{\text {max }}^{\mathrm{O}_{2}}, \mathrm{~K}_{\mathrm{M}}^{\mathrm{O}_{2}}$ and $D^{O_{2}}$ and the radiosensitivity parameters of tumor cells, especially those of the phase G1, also had a significant impact on the integral of tumor density.

In contrast, these results suggest that parameters associated with angiogenesis (in red), the division of healthy cells (in orange) and the response to irradiation of healthy and endothelial cells (in purple) were negligible for both the tumor density at $t=12$ weeks and the integral of tumor density endpoints.

The impact of parameters located to the right of the dashed lines in Fig. 4 was indistinguishable from the uncertainty due to the stochastic nature of the model.

To better illustrate the impact of the most important parameters of the model, TCP curves are presented in Fig. 5 for 6 different values of $T_{\text {tum }}, \alpha_{t u m G 1}, \alpha_{t u m G 2}$ and $p O_{2}^{\text {nec }}$ within the ranges specified in Table I. Reference values indicated in the same table were taken for the constant parameters. A 2 Gy fractionation administered every $24 \mathrm{~h}$ from Monday to Friday was considered. The experiment was repeated 10 times for each of the 21 computational tissues (a total of 210 simulations per curve).

It can be noticed that a small value of $T_{t u m}(85 \mathrm{~h})$ complicated tumor control. There seemed to be no major variation between TCP curves for the other values of $T_{t u m}$ (Fig. 5a). Tumor control probabilities increased with the radiosensitivity parameters of tumor cells in phases G2, $\alpha_{t u m G 2}$, and especially G1, $\alpha_{\text {tumG1 }}$, (Fig. 5c and b, respectively). Finally, the threshold of hypoxic death $\mathrm{pO}_{2}^{\text {nec }}$ (Fig. 5d) also had a significant effect on the TCP curves. Low values of this parameter, especially $0 \mathrm{mmHg}$ (no hypoxic death at all), considerably complicated tumor control.

\section{Simplification of the model}

The TCP curves obtained for the comprehensive model and the reduced versions are presented in Fig. 6. The reference parameter values presented in Table I and a 2 Gy fractionation were used for every repetition. As in Fig. 5, each TCP curve was built from $21 \times 10=210$ simulations.

The exclusion of angiogenesis from the model slightly complicated tumor control, whereas somewhat higher TCP values were obtained when the division of healthy cells was not considered. There seemed to be no major difference when the cycle arrest or the response to irradiation of healthy and endothelial cells were not included in the model (Fig. 6a).

The TCP curve obtained when angiogenesis, healthy cell division, cycle arrest and the response to irradiation of healthy and endothelial cells were simultaneously excluded is shown in Fig. 6b. No significant difference with respect to the reference TCP curve could be observed. It can be thus concluded that a simplified version of the model which considers neither angiogenesis nor healthy cell division nor cycle arrest nor the response to irradiation of healthy and endothelial cells (Fig. 7) is equivalent to the complete model integrating these mechanisms and sub-mechanism in terms of TCP. This reduced version includes only 18 parameters against 33 of the comprehensive model.

The TCP curves of the intermediate reduced versions excluding 2 and 3 mechanism or sub-mechanism combinations, as well as the values and the absolute and relative variations of the sigmoid constants $a$ and $b$ for every TCP curve can be found in Supplementary Fig. 3.

\section{Validation of the model. Biochemical recurrence prediction}

Results of the biochemical recurrence predictions are presented in Fig. 8. Mean ROC curves and confidence intervals obtained after 1000 repetitions for the 3 different sets of features are shown. The corresponding mean AUC values are indicated. Biochemical recurrence predictions based on the tumor area at $t=8$ weeks output of the comprehensive (AUC $=0.81 \pm 0.02$ ) and reduced (AUC $=0.82 \pm 0.02$ ) in silico models were significantly better than those obtained from the pre-treatment maximal tumor area and average ADC and T2w values $(\mathrm{AUC}=0.75 \pm 0.03)$. Furthermore, no major difference could be found between predictions made from the output of the comprehensive and the reduced versions of the model.

\section{Discussion}

The developed comprehensive model of tumor response to irradiation included the most relevant radiobiological mechanisms, which have been considered separately in previous multiscale approaches of the literature [29]-[32]. In particular, this is the first model simultaneously integrating, to our knowledge, mitotic catastrophe and cell cycle distribution, including checkpoints between phases G1/S and G2/M and a quiescence phase G0 affecting the radiosensitivity of tumor cells. This singularity offers a more realistic response to irradiation and opens doors to simulations considering cell cycle inhibitors or synchronizers [54]. In addition, this work introduced a novel approach of modeling angiogenesis, based on the calculation of dynamic VEGF distributions using a reaction-diffusion equation. Dynamic $\mathrm{pO}_{2}$ maps were obtained, as in the works of Espinoza et al. [43] and others [20], [55], using partial differential equations. The stochastic intrinsic radiosensitivity of tumor, endothelial and healthy cells was given by the linearquadratic formalism, adjusted to consider the influence of $\mathrm{O}_{2}$, as in [20], [24], [29]-[32].

\section{ACCEPTED MANUSCRIPT}




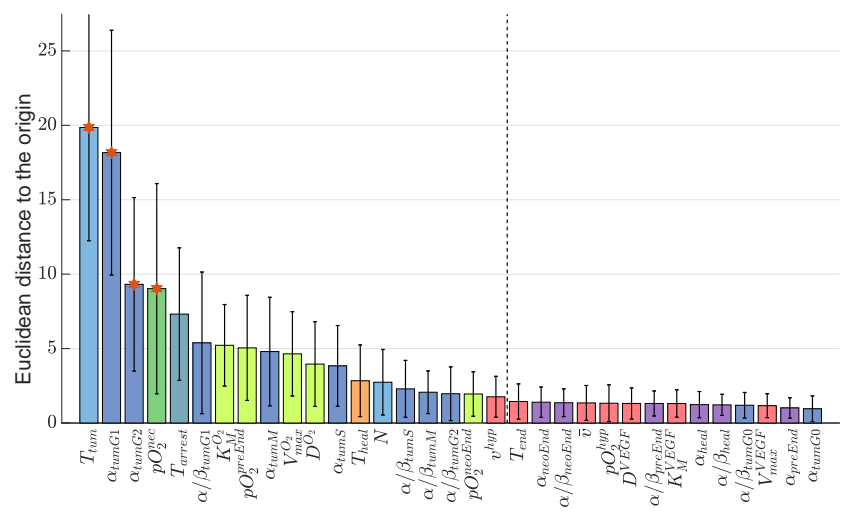

(a)

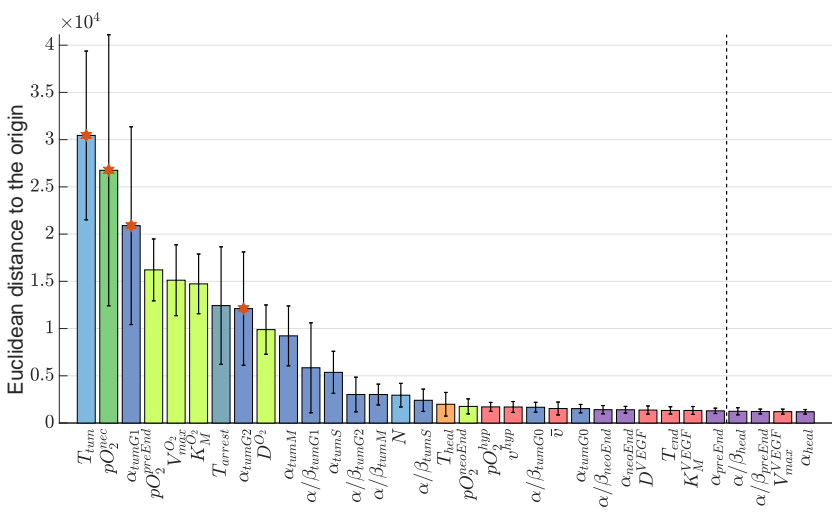

(b)

Fig. 4. Sensitivity analysis results. Euclidean distances to the origin, $S_{i}$, of the 33 parameters in descending order of importance taking as output: (a) the tumor density at $t=12$ weeks and (b) the integral of tumor density. Twenty-ones computational tissues obtained from prostate histological cuts of 7 patients were used for this analysis. Error bars represent the heterogeneity of $S_{i}$ among the different tissues. The same color code as in Table I is used. The effect of parameters to the right of the dashed lines is indistinguishable from the uncertainty caused by the intrinsic stochastic component of the model

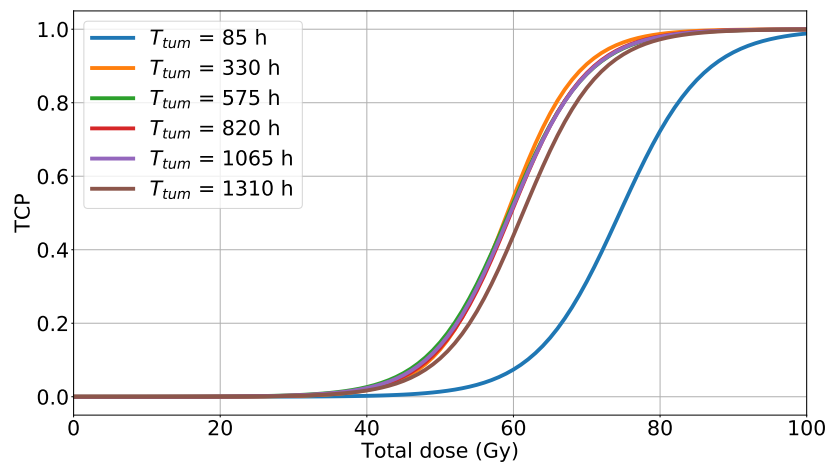

(a)

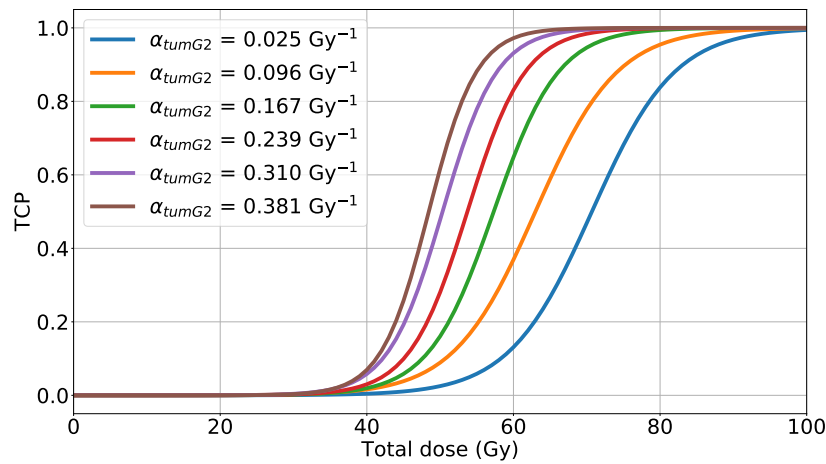

(c)

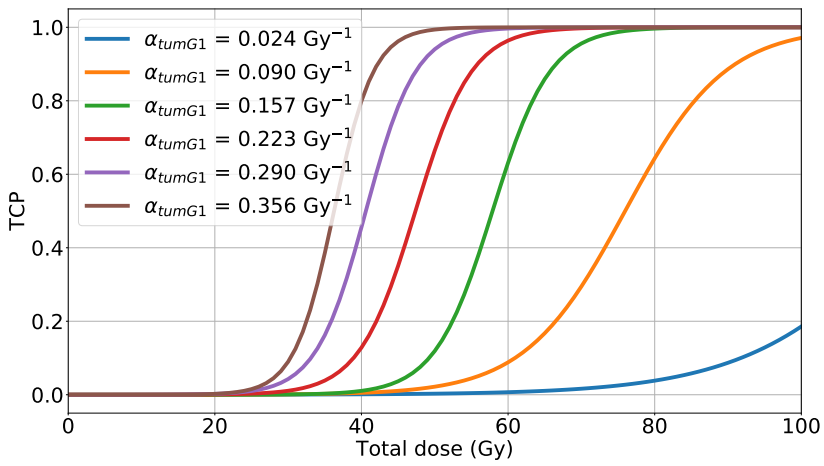

(b)

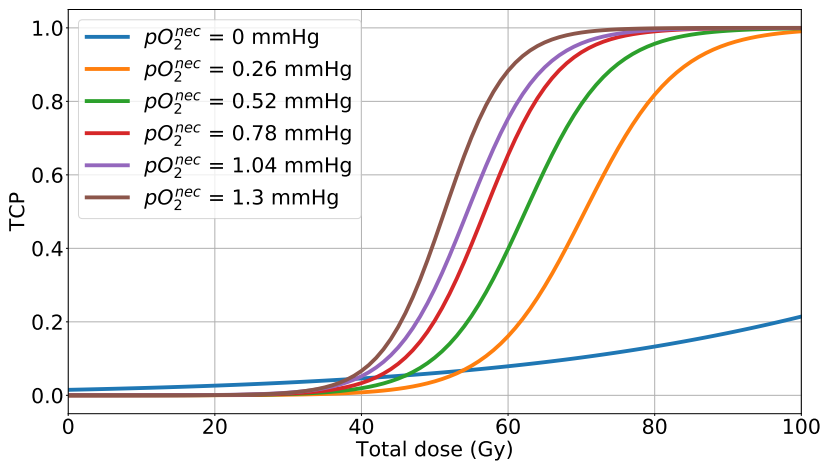

(d)

Fig. 5. TCP curves considering different values of (a) $T_{t u m}$, (b) $\alpha_{t u m G 1}$, (c) $\alpha_{t u m G 2}$ and (d) $p O_{2}^{\text {nec }}$, identified with a red star as the most important parameters in Fig. 4. A 2 Gy fractionation from Monday to Friday was used for every simulation. Six equidistant values within the ranges presented in Table I were considered for each studied factor. Reference values defined in the same table were used for the other parameters. A total of 210 simulations (10 repetitions $\times 21$ computational tissues) were performed to build each TCP curve.

The complexity of the model lay, thus, in the integration of all these different mathematical formalisms, including stochastic and deterministic, as well as continuous and discrete approaches using a single computational framework based on M2SL. Furthermore, it must be remarked that the considered radiobiological mechanisms take place at different timescales. Tumor, healthy and endothelial cell division and response to irradiation were simulated with a time-step in the order of hours while oxygenation and VEGF diffusion and consumption are much faster processes happening at the 


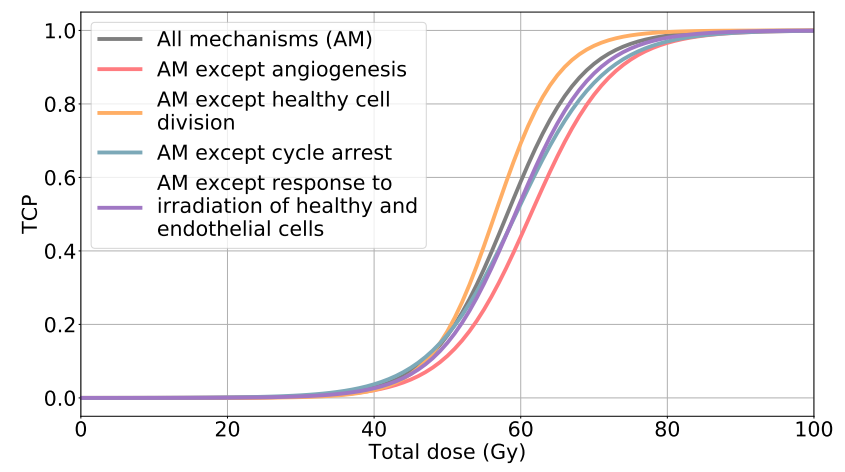

(a)

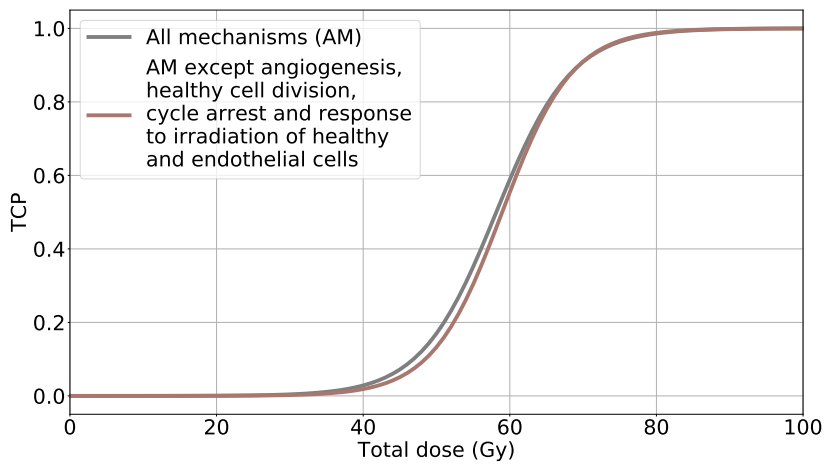

(b)

Fig. 6. TCP curves excluding (a) one and (b) 4 mechanisms or sub-mechanisms. A 2 Gy fractionation from Monday to Friday was simulated. The reference parameter values presented in Table I were considered. A total of 210 simulations (10 repetitions $\times 21$ computational tissues) were performed to build each TCP curve. The same color code as in Table I is used.

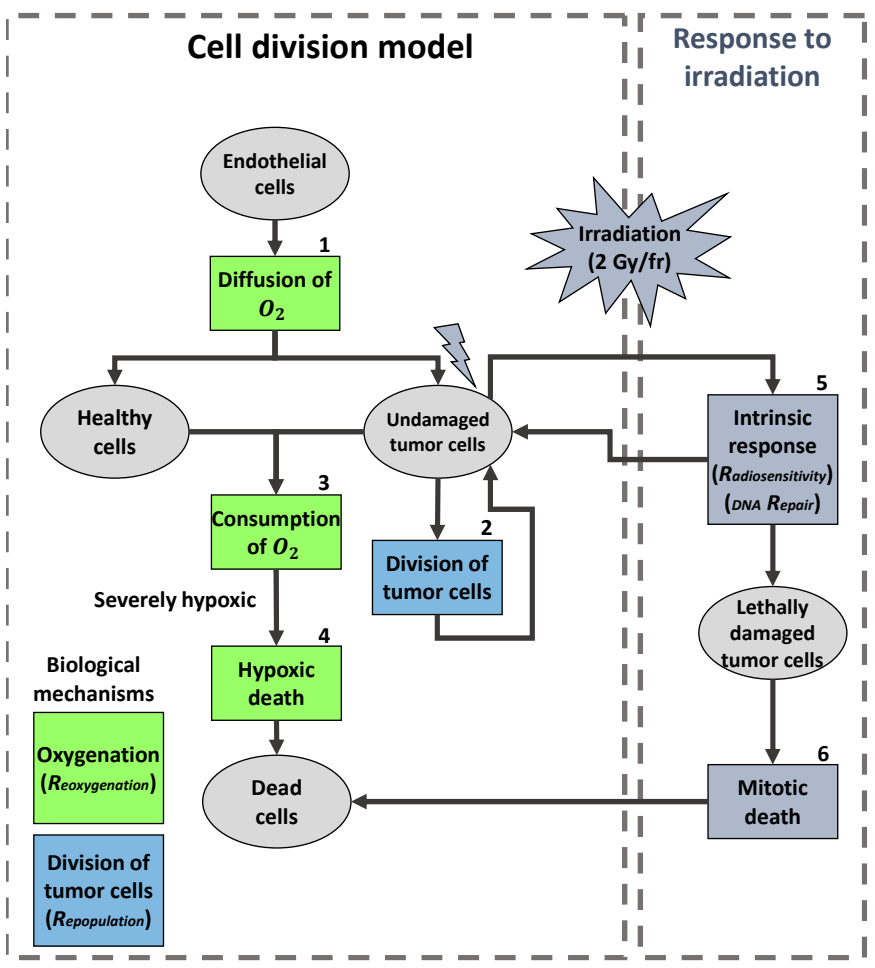

Fig. 7. Functional diagram of the reduced model. The different types of cells are represented with a gray ellipse. The different mechanisms are represented with a rectangle. The same color code as in Table I is used. Correspondences to the $5 \mathrm{R}$ 's are indicated in italics. Endothelial cells diffuse $\mathrm{O}_{2}$ (1). Tumor cells divide (2). Healthy and tumor cells consume $\mathrm{O}_{2}(3)$. As a result, if the $\mathrm{O}_{2}$ levels are extremely low, they become severely hypoxic, which provokes their death (4). According to their intrinsic response to radiation (5), tumor cells irradiated with a dose of 2 Gy per fraction resume their division, if their DNA can be repaired, or die at the next mitosis (mitotic death) (6), if the damage caused by irradiation is lethal.

scale of milliseconds. M2SL provided the tools to couple all the simulations at adapted frequencies without unnecessary computational burden.

The described model allowed in silico simulations of a complete radiotherapy protocol $(40 \times 2 \mathrm{~Gy})$. The obtained oxygenation maps (Fig. 2) showed that cells situated in the

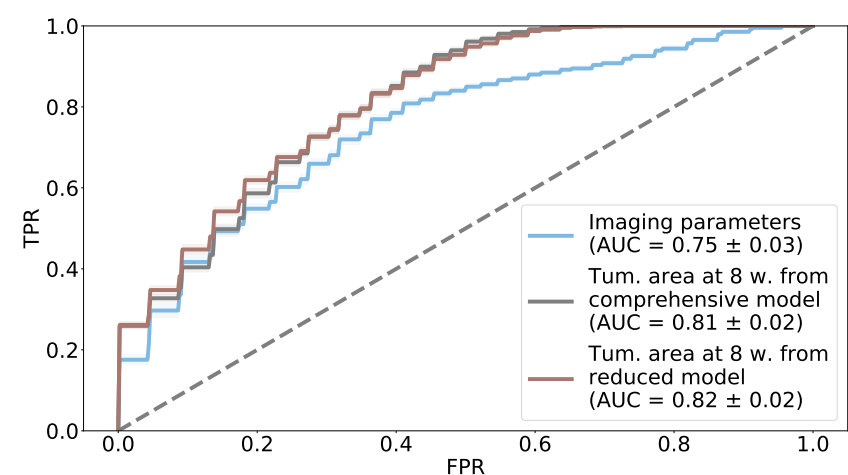

Fig. 8. ROC curves of biochemical recurrence predictions based on the maximal tumor area and average ADC and $\mathrm{T} 2 \mathrm{w}$ values observed before treatment (blue) and the tumor area at $t=8$ weeks outputs given by the comprehensive (gray) and reduced (brown) in silico models. Mean AUC values are indicated.

poorly vascularized tumor core presented low $\mathrm{pO}_{2}$ values. As $\mathrm{O}_{2}$ diffusion is limited to a certain range, vessels located in the tumor rim could not fully oxygenate too distant cells situated in the central region, which therefore developed hypoxia and, in severe cases, necrosis. Consequently, since VEGF is emitted by hypoxic cells, a higher concentration of this protein was found in the tumor core. All these results are in line with the literature consensus [15], [56], [57].

Furthermore, TCP curves could be obtained. It must be noticed that the tumor was supposed to be controlled when there were not any undamaged tumor cells in the tissue. This consideration might prove to be too strict in clinical cases as the immune system, which was not integrated in the model, may be capable of destroying small quantities of tumor cells remaining after a radiotherapy treatment, assuring tumor control [50].

As far as we can tell, this is the first work presenting the results of an exhaustive sensitivity analysis (71400 simulations) of an integrative in silico model of tumor response to irradiation. Other studies of the literature [20], [30], [31] have only performed partial approaches to individually evaluate the 
impact of certain mechanisms or parameters. Those one-ata-time analysis provide a preliminary view of the effect of a given factor with very few evaluations of the model. However, they prove to be insufficient and their results must be taken cautiously since these approaches cannot detect interactions between parameters. The Morris screening method used in this work provided qualitative information about the effect of the 33 parameters, including their interactions. This made it possible to rank, for the first time to our knowledge, all the parameters of an integrative model of tumor response to radiotherapy in order of importance. These results allowed to better assess the importance of the different radiobiological mechanisms and were used to reduce the dimension of the comprehensive model. Numerous examples of model reduction based on sensitivity analysis can be found in the biomedical literature [34], [35], [38].

Prostate histological cuts from 7 patients were used to initialize computational tissues for the sensitivity analysis and the model reduction simulations. These represent a large variability of tumor configurations. Results presented in this work are thus based on realistic tumor and vascular architectures.

The duration of the tumor cell cycle, $T_{t u m}$, was identified as the parameter having the highest impact on tumor density at $t$ $=12$ weeks and its integral. This is in agreement with previous partial results of the literature. Harting et al. [20] also noted that the mechanism of division of tumor cells had an important effect on TCP. Espinoza et al. [30] explored the effect of different values of the doubling time of tumor cells. This parameter, comparable to the $T_{\text {tum }}$ of our model, was found to have a significant influence on tumor cell density. Furthermore, the impact was higher when the value was decreased, which resulted in an increased division of tumor cells. All this is in consonance with results presented in our Fig. 5a in the form of TCP curves.

The impact of $T_{\text {tum }}$ can be interpreted as the sum of 2 contributions that apparently affect tumor density in opposite directions. On the one hand, it has an obvious effect on the proliferation of tumor cells. A low value of $T_{\text {tum }}$ results in a fast division of this kind of cells. On the other hand, as tumor cells die by mitotic catastrophe, $T_{\text {tum }}$ also impacts on their response to irradiation. A low value provokes a slow destruction of tumor cells.

Our parameters of dynamic oxygenation also had a significant effect on the studied outputs. In particular, hypoxic death had a great impact on tumor control. It can be suggested that, when this mechanism was not considered $\left(p O_{2}^{n e c}=0 \mathrm{mmHg}\right)$, extremely hypoxic cells, which are very radioresistant, did not die due to this lack of oxygen but had to be killed by irradiation, therefore at the expense of tumor control. The importance of oxygenation on tumor control was already illustrated by TCP curves presented by Harting et al. and PaulGilloteaux et al. [31].

In contrast, angiogenesis parameters had a negligible effect on both tumor density at $t=12$ weeks and its integral. When the whole angiogenesis mechanism was excluded, tumor was slightly more difficult to control. It can be hypothesized that the tissue was less oxygenated and, consequently, more radioresistant. However, the difference was not enormous. On this matter, Espinoza et al. showed that versions of their model considering an increased angiogenesis or no angiogenesis at all presented no significant differences in terms of tumor density.

The duration of the healthy cell cycle, $T_{\text {heal }}$, also had a negligible effect on the tumor density at $t=12$ weeks and its integral. When the whole mechanism was not considered, tumor control was slightly easier to achieve. It can be suggested that, in this case, the tissue contained more dead cells, which did not consume $\mathrm{O}_{2}$. Consequently, it was better oxygenated and, therefore somewhat less radioresistant. Nevertheless, the difference was not substantial. This is in agreement with the results of Espinoza et al. The analogous mechanism of resorption of dead tumor cells of their model was found to have no influence on tumor density.

The impact on tumor control of the 3 radiobiological mechanisms retained in the reduced model (oxygenation, division of tumor cells and their response to irradiation) has been largely reported in the literature [7]-[12]. In particular, the relationship between hypoxia and tumor response to irradiation has been widely discussed [56], [58]-[60]. Hypoxic regions, situated at a certain distance from blood vessels, are known to be more resistant to irradiation that well-oxygenated areas. To consider this heterogeneity, in this work, as in most of the previous in silico models [20], [30], we included, at the cell scale, the oxygen enhancement ratio (OER). This factor establishes a relation between the doses administered under hypoxic and aerobic conditions producing the same biological effect [15]. It can be expressed in alternative ways [24], [29], [31], [32].

Biochemical recurrence predictions made with the tumor area at $t=8$ weeks output of the comprehensive and the reduced versions of the model significantly improved those based on pre-treatment imaging parameters. These results show a potential application of the model as a useful tool in a clinical context. In addition, they corroborate the equivalence of the comprehensive and the reduced model, already suggested by the sensitivity analysis and demonstrated in terms of TCP curves, as no major difference was observed between the predictions made from the outputs of the 2 versions.

It must be remarked that this preliminary validation was performed taking the intermediate reference values of Table I for every parameter of the model, except for the highly influential hypoxic death threshold, $p \mathrm{O}_{2}^{n e c}$. Future model calibration, especially of the other parameters having an important impact on tumor area at $t=8$ weeks, should improve the quality of the predictions. Furthermore, an initial vascular density of $3.8 \%$ [31] was supposed for the 76 virtual tissues. Individual values for each sample deduced from PET images, may also lead to better biochemical recurrence predictions, as the sensitivity analysis showed that oxygenation parameters had an important impact on tumor regression.

As a first step, the presented results were obtained considering only a dose per fraction of 2 Gy, typically applied in prostate cancer. However, the flexible implementation of the model with MS2L will allow to simulate hypo-andhyperfractionated treatments [48], [61]-[63] in the future. Using optimization algorithms [64], [65], the total dose, the dose per fraction and the interval between sessions maximizing tumor control will be identified. 
This work presents several limitations which will be tackled in the future. Firstly, the comprehensive model intended to integrate the most relevant biological mechanisms of the literature, in particular those described by the 5 R's [3]. Nevertheless, some mechanisms which may also play a role on tumor regression, such as the immune response [50], were not included as only short-term evolution was considered. Furthermore, it must be remarked that the linear-quadratic equation, almost universally used to model the response to irradiation, may not be valid for every value of dose per fraction. The applicability of this formalism to high doses (more than 6 Gy) remains controversial [66].

Secondly, it must be borne in mind that the validity of the conclusions of the sensitivity analysis can only be guaranteed within the studied prostate-specific limit values of the parameters. The definition of appropriate ranges is, thus, a key aspect of the Morris method. Due to ethical or technical reasons, the vast majority of the parameters of cancer models have never been measured in vivo and, for many of them, a panoply of in vitro values exists [30], [31], [43], [46]-[49]. Ranges in this work were defined from maximum and minimum values of the literature and our radiobiological expertise. This should assure the significance of the obtained results. Nevertheless, to complement this approach, a more precise, but also more computationally expensive, sensitivity analysis using, for example, the Sobol method will be performed in the future on the reduced model, including only the most relevant parameters identified by the Morris analysis.

Thirdly, the results of the first validation of the model at the population scale need to be corroborated on an external cohort including a larger number of patients. Furthermore, although each mechanism has been previously considered in the literature, the model should still be validated at the microscopic and macroscopic scales. Several options to cope with this issue may be considered. Time-lapse imaging of prostate tumor cells cultured in vitro and irradiated with different fraction schedules can be used to carry out a rough validation of tumor cells division and response to irradiation at the microscopic scale. Cells can be tracked using detection strategies [67]. Nevertheless, a cell-based approach presents several limitations, the most important being the lack of consideration of realistic tumor microenvironment. At a macroscopic scale, several mechanisms can also be validated. Using longitudinal mpMRI [68], CBCT [24] and PET images [32] of irradiated patients, tumor volumes and cell densities can be obtained and compared with the results of in silico simulations. Additionally, PET images can be used to validate the oxygenation of the tissue [69], [70].

\section{CONCLUSION}

An original multiscale in silico model of tumor response to radiotherapy (33 parameters) integrating 5 radiobiological mechanisms (oxygenation, division of tumor cells, angiogenesis, division of healthy cells and phase-and-oxygen-dependent response to irradiation, considering cycle arrest and mitotic catastrophe) was developed. A thorough sensitivity analysis (71400 simulations) using the Morris method, applied for the first time to a whole integrative model of tumor response to radiotherapy, allowed to obtain a reduced version (18 parameters), equivalent in terms of tumor control probability and biochemical recurrence prediction. This simplified model included only 3 radiobiological mechanisms: oxygenation, given by a reaction-diffusion equation, division of tumor cells, considering their cycle, and cycle-phase-and-oxygendependent response to irradiation of tumor cells, based on the linear-quadratic formalism. Biochemical recurrence predictions obtained with the reduced model were significantly better than those performed from pre-treatment imaging parameters. The reduced model should still be thoroughly validated at the microscopic and macroscopic scales using experimental data. Other radiobiological mechanisms, such as the radio-induced tissue inflammation provoking neovascularization and immune cell recruitment, should be explored and potentially integrated. In the future, the model will be used to simulate different radiotherapy fractionation schedules (hypo/hyper fractionation), paving the way for the clinical optimization of patient-specific treatments.

\section{REFERENCES}

[1] J. Bernier, E. J. Hall, and A. Giaccia, "Radiation oncology: a century of achievements," Nat. Rev. Cancer, vol. 4, no. 9, pp. 737-747, 2004.

[2] A. V. D'Amico et al., "Biochemical Outcome After Radical Prostatectomy, External Beam Radiation Therapy, or Interstitial Radiation Therapy for Clinically Localized Prostate Cancer," JAMA, vol. 280, no. 11, pp. 969-974, 1998.

[3] R. J. Klement, "The influence of ketogenic therapy on the 5 R's of radiobiology," Int. J. Radiat. Biol., vol. 95, no. 4, pp. 394-407, 2019.

[4] M. Milosevic et al., "Tumor Hypoxia Predicts Biochemical Failure following Radiotherapy for Clinically Localized Prostate Cancer," Clin. Cancer Res., vol. 18, no. 7, pp. 2108 LP - 2114, 2012.

[5] A. Fraga et al., "Hypoxia and Prostate Cancer Aggressiveness: A Tale With Many Endings," Clin. Genitourin. Cancer, vol. 13, no. 4, pp. 295301, 2015.

[6] M. R. Horsman and J. Overgaard, "The impact of hypoxia and its modification of the outcome of radiotherapy," J. Radiat. Res., vol. 57, no. Suppl 1, pp. i90-i98, 2016.

[7] J. J. Kim and I. F. Tannock, "Repopulation of cancer cells during therapy: an important cause of treatment failure," Nat. Rev. Cancer, vol. 5, no. 7, pp. 516-525, 2005.

[8] H. R. Withers, J. M. G. Taylor, and B. Maciejewski, "The hazard of accelerated tumor clonogen repopulation during radiotherapy," Acta Oncol., vol. 27, no. 2, pp. 131-146, 1988.

[9] J. Z. Wang and X. A. Li, "Impact of tumor repopulation on radiotherapy planning," IJROBP, vol. 61, no. 1, pp. 220-227, 2005.

[10] B. Fertil and E. P. Malaise, "Intrinsic radiosensitivity of human cell lines is correlated with radioresponsiveness of human tumors: Analysis of 101 published survival curves," IJROBP, vol. 11, no. 9, pp. 1699$1707,1985$.

[11] L. E. Gerweck et al., "Tumor Cell Radiosensitivity Is a Major Determinant of Tumor Response to Radiation," Cancer Res., vol. 66, no. 17, pp. $8352-8355,2006$.

[12] C. M. L. West, "Intrinsic radiosensitivity as a predictor of patient response to radiotherapy," Brit. J. Radiol., vol. 68, no. 812, pp. 827837,1995

[13] W. Sinclair and R. Morton, "X-ray sensitiviy during the cell generation cycle of cultured Chinese hamster cells," Radiat. Res., vol. 29, pp. 450474, 1966.

[14] M. Castedo et al., "Cell death by mitotic catastrophe: a molecular definition," Oncogene, vol. 23, no. 16, pp. 2825-2837, 2004.

[15] E. J. Hall and A. J. Giaccia, Radiobiology for the Radiologist, 7th ed. Lippincott Williams \& Wilkins, 2012.

[16] M. Joiner and A. van der Kogel, Basic Clinical Radiobiology. London: Hodder Arnold, 2009

[17] M. Hadjicharalambous, P. A. Wijeratne, and V. Vavourakis, "From tumour perfusion to drug delivery and clinical translation of in silico cancer models," Methods, 2020.

\section{ACCEPTED MANUSCRIPT}


[18] S. Hamis, G. G. Powathil, and M. A. J. Chaplain, "Blackboard to Bedside: A Mathematical Modeling Bottom-Up Approach Toward Personalized Cancer Treatments," JCO Clin. Cancer Inform., no. 3, pp. $1-11,2019$.

[19] K. Borkenstein, S. Levegrün, and P. Peschke, "Modeling and Computer Simulations of Tumor Growth and Tumor Response to Radiotherapy," Radiat. Res., vol. 162, no. 1, pp. 71-83, 72004.

[20] C. Harting et al., "Single-cell-based computer simulation of the oxygendependent tumour response to irradiation," Phys. Med. Biol., vol. 52, no. 16, pp. 4775-4789, 2007.

[21] G. Powathil et al., "Mathematical modeling of brain tumors: effects of radiotherapy and chemotherapy." Phys. Med. Biol., vol. 52, no. 11, pp. 3291-3306, 2007.

[22] M. Kohandel et al., "Dynamics of tumor growth and combination of antiangiogenic and cytotoxic therapies." Phys. Med. Biol., vol. 52, no. 13, pp. 3665-3677, 2007.

[23] R. Rockne et al., "Predicting the efficacy of radiotherapy in individual glioblastoma patients in vivo: a mathematical modeling approach." Phys. Med. Biol., vol. 55, no. 12, pp. 3271-3285, 2010.

[24] A. Belfatto et al., "Modeling the Interplay Between Tumor Volume Regression and Oxygenation in Uterine Cervical Cancer During Radiotherapy Treatment," IEEE J. Biomed. Health Inform., vol. 20, no. 2, pp. 596-605, 2016.

[25] G. Lorenzo et al., "Mechanistic modelling of prostate-specific antigen dynamics shows potential for personalized prediction of radiation therapy outcome." J. R. Soc. Interface, vol. 16, no. 157, 2019.

[26] J. F. Fowler, "The linear-quadratic formula and progress in fractionated radiotherapy," Brit. J. Radiol., vol. 62, no. 740, pp. 679-694, 1989.

[27] D. J. Brenner, "The Linear-Quadratic Model Is an Appropriate Methodology for Determining Isoeffective Doses at Large Doses Per Fraction," Semin. Radiat. Oncol., vol. 18, no. 4, pp. 234-239, 2008.

[28] D. Brand and J. Yarnold, "The Linear-Quadratic Model and Implications for Fractionation," Clin. Oncol., vol. 31, no. 10, pp. 673-677, 2019.

[29] B. Titz and R. Jeraj, "An imaging-based tumour growth and treatment response model: investigating the effect of tumour oxygenation on radiation therapy response," Phys. Med. Biol., vol. 53(17), pp. 44714488, 2008.

[30] I. Espinoza, P. Peschke, and C. P. Karger, "A voxel-based multiscale model to simulate the radiation response of hypoxic tumor," Med. Phys., vol. 42.1, pp. 90-102, 2015.

[31] P. Paul-Gilloteaux et al., "Optimizing radiotherapy protocols using computer automata to model tumour cell death as a function of oxygen diffusion processes," Sci. Rep., vol. 7, 2017.

[32] S. Apeke et al., "Multi-Scale Modeling and Oxygen Impact on Tumor Temporal Evolution: Application on Rectal Cancer During Radiotherapy," IEEE Trans. Med. Imag., vol. 37, no. 4, pp. 871-880, 2018.

[33] A. I. Hernandez et al., "A multiformalism and multiresolution modelling environment: application to the cardiovascular system and its regulation," Phil. Trans. Math. Phys. Eng. Sci., vol. 367(1908), pp. 4923-4940, 2009.

[34] A. Saltelli et al., Global Sensitivity Analysis. The Primer, 12008.

[35] Z. Hu and P. Shi, "Sensitivity Analysis for Biomedical Models," IEEE Trans. Med. Imag., vol. 29, no. 11, pp. 1870-1881, 2010.

[36] I. M. Sobol', "Sensitivity estmiates for non linear mathematical models," Math. Modelling Comput. Experiments, vol. 1, pp. 407-414, 1993.

[37] M. Morris, "Factorial sampling plans for preliminary computational experiments," Technometrics, vol. 33.2, pp. 161-174, 1991.

[38] G. S. Papoutsoglou and C. Balas, "Estimation of Neoplasia-Related Biological Parameters Through Modeling and Sensitivity Analysis of Optical Molecular Imaging Data," IEEE Trans. Biomed. Eng., vol. 60, no. 5, pp. 1241-1249, 2013.

[39] D. Ojeda et al., "Sensitivity Analysis and Parameter Estimation of a Coronary Circulation Model for Triple-Vessel Disease," IEEE Trans. Biomed. Eng., vol. 61, no. 4, pp. 1208-1219, 2014.

[40] — , "Sensitivity Analysis of Vagus Nerve Stimulation Parameters on Acute Cardiac Autonomic Responses: Chronotropic, Inotropic and Dromotropic Effects," PLOS ONE, vol. 11, no. 9, 2016.

[41] C. Sosa Marrero et al., "Sensitivity Analysis Of An In Silico Model Of Tumor Growth And Radiation Response," in IEEE ISBI 2019, pp. $1497-1500$.

[42] D. Ojeda, "Multi-resolution physiological modeling for the analysis of cardiovascular pathologies," Ph.D. dissertation, Univ. Rennes 1, 2013.

[43] I. Espinoza, P. Peschke, and C. P. Karger, "A model to simulate the oxygen distribution in hypoxic tumors for different vascular architectures," Med. Phys., vol. 40, 2013.

[44] F. Campolongo et al., "Enhancing the Morris method," in SAMO 2004.
[45] V. A. Potiron et al., "Improved Functionality of the Vasculature during Conventionally Fractionated Radiation Therapy of Prostate Cancer," PLOS ONE, vol. 8, no. 12, pp. 1-10, 2014.

[46] M. W. Dewhirst et al., "Perivascular Oxygen Tensions in a Transplantable Mammary Tumor Growing in a Dorsal Flap Window Chamber," Radiat. Res., vol. 130, no. 2, pp. 171-182, 1992.

[47] A. Haworth et al., "Validation of a radiobiological model for low-doserate prostate boost focal therapy treatment planning," Brachytherapy, vol. 12(6), pp. 628-636, 2013.

[48] D. J. Brenner and E. J. Hall, "Hypofractionation in prostate cancer radiotherapy," Transl. Cancer Res., vol. 7, no. 6, 2018.

[49] D. J. Carlson et al., "Comparison of in vitro and in vivo ratios for prostate cancer," Phys. Med. Biol., vol. 49, no. 19, pp. 4477-4491, 2004.

[50] P. Mayles, A. Nahum, and J. C. Rosenwald, Handbook of Radiotherapy Physics, 1st ed. CRC Press, 2007.

[51] K. Gnep et al., "Haralick textural features on T2-weighted MRI are associated with biochemical recurrence following radiotherapy for peripheral zone prostate cancer," J. Magn. Reson. Imaging, vol. 45, pp. 103-117, 2017.

[52] M. C. Abramowitz et al., "The Phoenix definition of biochemical failure predicts for overall survival in patients with prostate cancer." Cancer, vol. 112 , no. 1, pp. 55-60, 2008.

[53] J. T. Kwak et al., "Prostate Cancer: A Correlative Study of Multiparametric MR Imaging and Digital Histopathology.” Radiology, vol. 285, no. 1, pp. 147-156, 2017.

[54] K. Otani et al., "Cell-cycle-controlled radiation therapy was effective for treating a murine malignant melanoma cell line in vitro and in vivo," Sci. Rep., vol. 6, no. 1, p. 30689, 2016.

[55] A. Daşu, I. Toma-Daşu, and M. Karlsson, "Theoretical simulation of tumour oxygenation and results from acute and chronic hypoxia," Phys. Med. Biol., vol. 48, no. 17, pp. 2829-2842, 2003.

[56] R. H. Thomlinson and L. H. Gray, "The histological structure of some human lung cancers and the possible implications for radiotherapy." $\mathrm{Br}$. J. Cancer, vol. 9, no. 4, pp. 539-549, 1955.

[57] B. Bao et al., "Hypoxia Induced Aggressiveness of Prostate Cancer Cells Is Linked with Deregulated Expression of VEGF, IL-6 and miRNAs That Are Attenuated by CDF," PLOS ONE, vol. 7, no. 8, pp. 1-11, 2012.

[58] L. H. Gray et al., "The concentration of oxygen dissolved in tissues at the time of irradiation as a factor in radiotherapy." Brit. J. Radiol., vol. 26, no. 312, pp. 638-648, 1953.

[59] E. A. Wright and P. Howards-Flanders, "The influence of oxygen on the radiosensitivity of mammalian tissues." Acta Radiol., vol. 48, no. 1, pp. 26-32, 1957.

[60] B. Palcic and L. D. Skarsgard, "Reduced Oxygen Enhancement Ratio at Low Doses of Ionizing Radiation," Radiat. Res., vol. 100, no. 2, pp. 328-339, 1984.

[61] S. Arcangeli et al., "Updated Results and Patterns of Failure in a Randomized Hypofractionation Trial for High-risk Prostate Cancer," IJROBP, vol. 84, no. 5, pp. 1172-1178, 2012.

[62] D. Dearnaley et al., "Conventional versus hypofractionated high-dose intensity-modulated radiotherapy for prostate cancer: 5-year outcomes of the randomised, non-inferiority, phase $3 \mathrm{CHHiP}$ trial," Lancet Oncol., vol. 17, no. 8, pp. 1047-1060, 2016.

[63] L. Incrocci et al., "Hypofractionated versus conventionally fractionated radiotherapy for patients with localised prostate cancer (HYPRO): final efficacy results from a randomised, multicentre, open-label, phase 3 trial," Lancet Oncol., vol. 17, no. 8, pp. 1061-1069, 2016.

[64] A. Gago-Arias et al., "Modelling radiation-induced cell death and tumour re-oxygenation: local versus global and instant versus delayed cell death," Phys. Med. Biol., vol. 61, no. 3, pp. 1204-1216, 2016.

[65] K. Leder et al., "Mathematical Modeling of PDGF-Driven Glioblastoma Reveals Optimized Radiation Dosing Schedules," Cell, vol. 156, no. 3, pp. 603-616, 2014.

[66] J. P. Kirkpatrick, J. J. Meyer, and L. B. Marks, "The Linear-Quadratic Model Is Inappropriate to Model High Dose per Fraction Effects in Radiosurgery," Semin. Radiat. Oncol., vol. 18, no. 4, pp. 240-243, 2008.

[67] V. Ulman et al., "An Objective Comparison of Cell Tracking Algorithms," Nat. Methods, vol. 14, 2017.

[68] T. Roque et al., "A DCE-MRI Driven 3-D Reaction-Diffusion Model of Solid Tumor Growth," IEEE Trans. Med. Imag., vol. 37, no. 3, pp. 724-732, 2018.

[69] D. Warren and M. Partridge, "The role of necrosis, acute hypoxia and chronic hypoxia in 18F-FMISO PET image contrast: A computational modelling study," Phys. Med. Biol., vol. 61, pp. 8596-8624, 122016.

[70] V. Adhikarla and R. Jeraj, "An imaging-based computational model for simulating angiogenesis and tumour oxygenation dynamics," Phys. Med. Biol., vol. 61, no. 10, pp. 3885-3902, 2016. 